\title{
Zur Casuistik der 0varialschwangerschaft.
}

Von

Otto Spiegelberg.

Als directe Beweise für das Vorhandensein einer Eierstocksschwangerschaft sind von Cohnstein jüngst in diesem Archiv (Bd. XII, S. 367) aufgestellt: 1) das Fehlen des bezüglichen Eierstockes; 2) Verbindung des Fruchtsäckes mit der Gebärmutter durch das verlängerte und verdickte Ligamentum ovarii, mit Umschliessung des Sackes von beiden Blättern des breiten Bandes; 3) lamellöser Bau der Umhüllung und Cylinderepithel der Innenfläche; unmittelbarer Uebergang der Faserzüge der Albuginea in die Wandung des Fruchtsackes; 4) in unmittelbarer Nähe der Eihöhle Bestandtheile von Ovarialgewebe. - Ein nothwendiger Befund ist aber nicht jeder von diesen; ja einzelne nicht einmal wahrscheinliche. Ein zum Fruchthalter gewordener Eierstock ist topographisch ein Eierstockstumor, wie ein Kystom, und doch wird Niemand behaupten können, dass eine solche Geschwulst von den beiden Blättern des Ligamentum latum umschlossen sei. Ein primär vorhandenes Cylinderepithel der Innenfläche des Eisackes wird wohl mit weiterem Wachsthume des letzteren und mit der Entwickelung der Placenta verschwinden; ein lamellöser Bau der Hülle von Zufälligkeiten abhängen, da er aus der Structur des Ovarialstromas nicht mit Nothwendigkeit sich ergiebt, und bei den verschiedensten Formen der extrauterinen Schwangerschaft vorhanden sein kann; von einem Uebergange der Faserzüge der Albuginea in die Fruchtsackwand kann schliesslich nur die Rede sein, wo noch Reste des Ovarium und somit seiner Albuginea als intact nachzuweisen und in ihrem Verlaufe zu verfolgen sind. Somit bleiben, als für die Annahme des ovariellen Charakters 
eines Fruchtsackes nothwendige Postulate, nur das Fehlen des Eierstockes einer Seite; Eierstockselemente in der Wand des Sackes, also Verbrauch jenes Organes zur Bildung des letzteren; Verbindung der Cyste mit der Gebärmutter durch das Eierstocksband; und schliesslich, was Cohnste in nicht anführte, Nichtbetheiligung der Eileiter an der Bildung des Fruchthalters, ein topographisches Verhalten auf der betroffenen Seite gleich oder ähnlich dem bei grossen Eierstockstumoren vorkommenden.

Legt man diesen Maasstab nun an die, als thatsächliche oder nicht zu bezweifelnde Fälle von Ovarialschwangerschaft beschriebenen an, wie sie Schröder in der 5. Auflage seines Lehrbuches, S. 407, anführt (es sind die von Willigk, Hein, Haselberg, Martyn, Gusserow-Hess, Kiwisch, Hecker, Wright, Schwenningerl)), denen sich der exquisite von Walter ${ }^{2}$ ) anschliesst - so muss von ihnen der Ha selberg' sche nach der vorliegenden Schilderung jedenfalls ausfallen; denn wenn auch in der nächisten Nähe der kleinen Eihöhle (der Fötus war $3 \mathrm{Cm}$. lang) ein Corpus luteum sich fand, so ist doch das Vorhandensein der Tube und ihr Verhalten nicht nachgewiesen. Auch an den übrigen Fällen, mit dem Walter'schen somit neun im Ganzen, liesse sich wegen der nicht immer genügenden Beschreibung noch mancher Zweifel erheben, wie das auch schon Hecker (Monatsschrift für Geburtskunde, XIII) ausdrückte; doch kann man sie immerhin alle gelten lassen.

Von den weiteren, durch Cohnstein (l. c. S. 368) der obigen Liste noch angereihten Fällen steht jedoch keiner der Kritik. Am ehesten hätte noch der Reymond'sche (Annales de Gynécologie II, 1874, S. 67) Anspruch, als ovarielle Schwangerschaft zu gelten, denn es fehlte das Ovarium und es bestand die Sackwand angeblich aus Eierstocksgewebe. Diese Behauptung hat freilich keinen Werth, wenn auch Cylinderepithel auf der Innenfläche sich fand, weil nicht angegeben, dass drüsige ovarielle Elemente vorhanden waren. Und vollends zweifelhaft wird die Sache dadurch, dass vom Ligamentum ovarii keine Rede ist und dass vom äusseren Ende der Tube gesagt wird: ,l'extremité externe de la trompe aboutit à une poche, qui constitue les deux tiers de la tumeur." (S. 70) - es wird sich also wohl um die sogenannte

1) Die Quellen s. bei Schröder.

2) Monatsschrift für Geburtskunde, XVIII, S. 171. 
tubo-ovarielle Form gehandelt haben. - Auch der von Guichard in derselben Zeitschrift, III, 1875 , S. 224 , als varieté ovarique beschriebene und jüngst wieder in den Archives de Tocologie, Aug. 1877, S. 489 reproducirte Fall ist nur eine epiovarielle Form, da das Ovarium erhalten, wenn auch plattgedrückt und mit der Wand des Fruchtsackes verbunden war.

Sonach verbleiben in der Literatur nur neun ganz oder nahezu ganz zuverlässige Fälle von Eierstocksschwangerschaft verzeichnet.')

Als zehnten füge ich die folgende Beobachtung an, welche auch in therapeutischer Beziehung einiges Interesse bietet.

Bis auf vage Schmerzen ungestörter Verlauf der Schwangerschaft. Am normalen Ende derselben Usur des Fruchtsackes und Peritonitis. Gastrotomie bei hoffnungslosem Zustande der Mutter. Das ausgetragene Kind erhalten.

Die Droschkenkutscherfrau F. hierselbst meldete sich im Juli 1876 in der Poliklinik. Sie ist 36 Jahre alt, mit 20 Jahren zuerst menstruirt, hat vor neun Jahren ein Mal regelmässig geboren. Sie leidet seit mehreren Jahren an Schmerz in der linken Unterbauchgegend, welcher in der letzten Zeit und mit dem Ausbleiben der Regel stärker geworden. Am Ende Mai bis Anfang Juni waren die Menses zum letzten Male erschienen. - Die Untersuchung ergab mässige Schwellung des Uterus, nahe der rechten Seite des unteren $\mathbf{A b}$ schnittes des Corpus und des Collum lag eine ungefähr kleinapfelgrosse Geschwulst, welche die hintere Partie der Beckenhöhle einnahm; sie hatte unebene verwaschene Umrisse und liess sich in ihrer Umgebung nicht verschieben; imponirte als entzündlicher Knoten unter der hinteren Platte des breiten Bandes. Dabei lag die Wahrscheinlichkeit bestehender uteriner Schwangerschaft sehr nahe. Wir verloren nun die Kranke aus den Augen, bis am Abend des 5. März vorigen Jahres unsere Hülfe wieder gesucht wurde. Es ward berichtet, dass die Schwangerschaft bis vor einigen Tagen ohne weitere Störung verlaufen sei, dass vor vier Tagen, am erwarteten richtigen Ende derselben sich Wehen gezeigt hätten, mit ihnen aber auch Zeichen von Unterleibsentzündung; diese letzteren seien seit dem vorigen Tage (4. März) stärker geworden, es habe sich, bei seit vier Tagen bestehender Verstopfung, Erbrechen eingestellt, welches am laufenden Tage mehrmals kothige Beschaffenheit gezeigt habe.

Herr Dr. W i en er, Assistenzarzt der Poliklinik, sah die Kranke nach 6 Uhr Abends. Er fand hochgradig entwickelte diffuse Peritonitis; Puls klein und elend, 148; häufig Erbrechen grüner dünner,

1) Einige bei Thieren gesehene s. bei Franck, Handbuch der thierärztlichen Geburtshülfe, 1876, S. 206. 
nicht kothiger Massen. Der enorm meteoristische, höchst empfindliche Bauch liess eine ausgiebige Palpation nicht zu; an der rechten Seite jedoch war kräftiger Fötalpuls deutlich zu hören. Die vaginale Untersuchung zeigte den Uterus median gelegen, etwas in die Höhe gezogen, vom Umfange eines zwei bis drei Monate schwangeren; Hals wie innerer Muttermund waren bequem durchgängig, so dass der Zeigefinger tief in das Corpus vordringen konnte; die Innenfläche war mit einem dicken, weichen, zum Theil schon abgelösten, leicht abstreif baren Schleimhautpolster (Decidua) bedeckt. In der linken Seite des Beckeneinganges, der vorderen Wand näher als der hinteren, ballotirte der harte grosse Fruchtkopf. (Grosse Dosen Morphiums.)

Den vorstehenden Befund und die damit gegebene Diagnose auf Peritonitis, Extrauterinschwangerschaft, lebende Frucht, Expulsivbestrebungen, konnte ich, $8 \mathrm{Uhr}$ bei der Kranken angelangt, nur bestätigen. Der Zustand war ein hoffnungsloser für die Mutter; es galt, das Kind zu erhalten; lag ja auch in der Eröffnung, Entleerung und Reinigung der Bauchhöhle die einzige Möglichkeit, noch etwas für die Mutter zu thun. Ihre Einwilligung zur Gastrotomie gab diese gerne.

Die Operation, ausgeführt in der engen Kellerwohnung, auf dem niedrigen weichen Bette, beleuchtet nur durch eine kleine Petroleumlampe, und unterstützt nur durch die Hebamme und den, gleichzeitig die Chloroformnarkose überwachenden Herrn Dr. Wiener, war eine mühselige. Ein vom Nabel bis nahe an die Schamfuge geführter Schnitt entleerte eine grosse Masse dünnen, janchigen, blutig tingirten Eiters. Der Fruchtsack lag frei zu Tage; er erschien dünnwandig, mit weiten Gefässen durchzogen, der Kindskörper schimmerte durch. Als ich die Hülle nun incidirte, erfolgte sehr starke Blutung, und bei der Erweiterung der Oeffnung zeigte es sich, dass die Placenta in grosser Länge getroffen war. Fruchtwasser war nur ganz spärlich noch vorhanden. Rasch zog ich das mit dem Rïcken nach rechts gelagerte Kind an den Unterextremitäten zu Tage; es erschien leicht asphyktisch, wurde rasch abgenabelt und Dr. Wiener übergeben, unter dessen Händen es bald laut schrie. Unterdess comprimirte ich mit beiden Händen die Wundränder des Fruchtsackes und. hemmte so momentan die Blutung aus ihnen. Als nun die Hebamme das Kind übernommen hatte und mir die Hülfe des Dr. Wiener wieder gesichert war, zeigte es sich, dass der vorliegende breite Rand der Placenta durchtrennt war, diese selbst in weiter Ausdehnung den Sack auskleidete. Den abgeschnittenen Lappen löste ich ab, zog den Sack ohne Hinderniss handbreit aus der Bauchwunde vor, und stillte durch Unstechung zunächst die Blutung aus dem Rande, an welchem der Kuchenlappen abgetrennt war. Nicht so leicht gelang dies an dem anderen Rande, von welchem aus der Kuchen sich in die Cyste ausbreitete; ein glücklicher Zufall war es eben, dass der Sack sich vorziehen liess, so genügend entfernt vom Schnittrande in mehreren Partien zugleich mit der anhaftenden Placenta durch- 
und umstochen werden konnte; eine Gesammtligatur sicherte dann das Erreichte noch weiter. An eine Exstirpation des Sackes war aber nicht zu denken, nach der rechten Seite und an der hinteren Fläche hing er den Därmen an. Beim Eingehen mit der Hand entdeckte ich denn auch an seiner, früher oberen, jetzt nahe dem oberen Bauchwundwinkel gebrachten Kuppe, einen kleinen Defect, die Stelle des spontanen Durchbruches. - Nachdem die Bauchhö́hle, so viel es bei dem sehr spärlich vorhandenen Hülfsmateriale anging, gereinigt war, befestigte ich die vorgezogene Cystenpartie mittels zwei durch ihre Masse und die Bauchwand geführter Ligaturen in der Wunde, vereinigte diese in der Umgebung der Cyste; die Nabelschnur und ein eingelegter Katheter hielten den Zugang zu letzterer offen.

Die Entbundene war durch den nicht geringen Blutverlust so verfallen, dass wir fürchteten, sie würde vor der Vollendung der Operation sterben. Sie erholte sich unter verabreichten Reizmitteln wohl etwas aus dem tiefen Collaps, starb aber noch im Laufe der Nacht.

Das Kind, ein kräftiges wohlgebildetes Mädehen, gedieh bei künstlicher Ernährung zunächst vortrefflich. Doch erlag es, drei Monate alt, dem Loose der meisten dieser mutterlosen Geschöpfe der Armen, ging an Atrophie zu Grunde.

Eine volle Obduction dex Mutter ward nicht gestattet; wir durften nur den Fruchtsack entfernen. Abgesehen von zahlreichen dïnnen und fadenförmigen Adhäsionen an der Serosa parietalis der rechten Seite, an der Gegend oberhalb des Nabels, an den Mesenterien, war der Sack innig verbunden nur mit den rechtsseitigen Beckenorganen und dem Coecum. Er wurde mit Uterus und Adnexen, sowie mit dem Coecum excidirt und dem pathologischen Institute übergeben. Von dort erfolgte folgender Bericht:

„Der Uterus ist vergrössert, $14 \mathrm{Cm}$. lang, am Grunde $10 \mathrm{Cm}$. breit, die Wand hier $1^{1 / 2} \mathrm{Cm}$. dick; der Hals hat $5 \mathrm{Cm}$. Länge. Die Wand sehr blutreich. Die Körperschleimhaut gesehwellt, rauh und fetzig, die oberste Schicht sehr locker und leicht abzuschaben. Halsschleimhaut unversehrt, verdickt und succulent.

„Auf beiden Seiten geht die Tube regelmässig ab. Links ist dieselbe in $7 \mathrm{Cm}$. Länge intact erhalten, das äussere Ende verliert sich in pseudomembranösen Anhängen auf dern hinteren Blatte des linken Ligamentum latum, in denen auch das linke Ovarium eingebettet ist. Dieses besitzt gewöhnliche Grösse, erscheint derb, mit vielen narbigen Einkerbungen, seine Schnittfäche ist blass, Reste von gelben Körpern sind nicht vorhanden.

„Auf der rechten Seite befindet sich, mit dem Uterus durch das Ligamentum ovarii und die Ala vespertilionis verbunden, ein Sack, der in seinem zusammengefallenen Zustande noch $10 \mathrm{Cm}$. Durchmesser hat. An seiner Vorderseite hängt er mittels einer breiten 
dicken Adhäsion, in welcher einige grosse Gefässe verlaufen, mit dem Coecum zusammen. Die rechte Tube läuft zunächst in fast $10 \mathrm{Cm}$. Länge im oberen Rande des verdichteten und succulenten Ligamentum latum, geht dann auf den Sack über; auf diesem lässt sie sich noch in $22 \mathrm{Cm}$. Länge verfolgen, und zwar $12 \mathrm{Cm}$. mit deutlichem Lumen, die weiteren $10 \mathrm{Cm}$. als schmales plattes Band, das sich dann in der Aussenfläche des Sackes verliert (Fimbria ovarica). $25 \mathrm{Cm}$. entfernt von der Stelle, an der der Eileiter an letzteren herantritt, findet sich eine platte Blase von $21 / 2$ resp. $2 \mathrm{Cm}$. Durchmesser, gelblich trüber Farbe, mit $1 \mathrm{Cm}$. langem dünnen Stiele und schmierigem Inhalte (Hydatide). Im Uebrigen ist die Aussenfläche des Sackes leicht höckerig, und an der rechten Seite und den oberen Partien zahlreich mit gefässreichen zerrissenen Adhärenzen besetzt.

„Das Ligamentum ovarii dieser Seite ist verdickt und enthält mehrere grosse Gefässe. In seiner Verlängerung liegt an der medialen Seite der unteren Partie des Sackes in dessen Wand ein weisser höckeriger derber Körper von nahezu Wallnussgrösse, in diesem selbst ein kleines Bläschen (Follikel) und eine kirschgrosse Cyste mit ockergelber schmieriger, von zahlreichen feinen Härchen durchsetzter Masse. Dieses Gebilde (Dermoidkystom) verliert sich in der Substanz der Sackwand obne bestimmte Grenze.

„Die Cystenwand ist an ihrer oberen Partie äusserst dünn und zart; sie besteht aus zwei Blättern, einem äusseren derben und dickeren und einem inneren ganz feinen, welche sich leicht von einander trennen lassen. Der grösste Theil der inneren Wand aber ist zu Placentargewebe umgewandelt, welches nach unten am dicksten ist, nach oben dünner wird, um sich schliesslich ganz allmälig in die zarte schleimhautähnliche innere Lamelle der oben gelegenen Sackpartie zu verlieren. Dieses Placentargewebe reicht überall bis dicht unter die äusserste Hülle der Cyste heran. Der Innenfläche liegt das Amnion noch auf. An dem untersten und lateralen Theile des Sackes inserirt die noch $45 \mathrm{Cm}$. lange Nabelschnur; sie zeigt keine Besonderheiten und löst sich in gewöhnlicher Weise in ihre, in die Placenta einstrahlenden Gefässe auf."

Der Beweis, dass der rechte Eierstock in vorliegendem Falle zum Fruchtsacke geworden, ist durch vorstehenden Befund in klarster Weise erbracht: Es fehlte der rechte Eierstock; in der Wand des Fruchtsackes wurden exquisite ovarielle Elemente aufgefunden; derselbe hatte die nothwendige Verbindung mit dem Uterus durch das Eierstocksband, und die Tube verhielt sich ganz so, wie man es bei grossen Ovarialtumoren so häufig sieht; sie war mit dem heranwachsenden Sacke, dem sie früh schon durch die Mesosalpinx adhärent geworden, enorm in die Länge gezogen, ihr Fimbrialende noch deutlich. 
Ueber die Gastrotomie selbst will ich weitere Worte nicht verlieren. Diese Frage habe ich in meinem Lehrbuche genügend abgehandelt, und jüngst hat sie Gusserow (dieses Archiv, Bd. XII, S. 75) in seiner klaren Weise wieder erörtert. Nur die schnelle Ausfuihrung der Operation hat im vorliegenden Falle das Kind gerettet. - Ein besonderes Interesse bietet die Complication, dass die Placenta in den Schnitt fiel. Wo ein solches Ereigniss bislang erwähnt wurde (Koeberle, s. bei Gusserow, S. 86; Hall Davis in Lancet, July 14, 1877 und Obstetrical Journal of Great Britain, Aug. 1877, S. 347), war die Frucht abgestorben, und es fehlte demgemäss zunächst Blutung oder war augenscheinlich ganz gering; doch wurde durch Nachblutung noch vor Schluss der Wunde Davis genöthigt, den Sack mit Eisenchlorid auszuwaschen, und sicher hatte diese Blutung das tödtliche Ende beschleunigt. Dass dagegen bei lebendem Fötus eine sehr schwer zu bemeisternde Blutung eintreten muss, hat schon Gusserow (l. c. S. 86) bemerkt und die vorliegende Beobachtung hat diese Meinung bestätigt. Da nun von einer Ablösung und Entfernung der ganzen Placenta in der Regel nicht die Rede sein kann, so wird bei derartiger, vorher ja nicht $\mathrm{zu}$ bestimmender Sachlage nichts Anderes zu thun bleiben, als was ich that: die durchschnittene Placentarpartie zu umstechen, dann mit Liquor ferri zu durchtränken und nach aussen zu leiten. Aber so schnell man alles das auch bewerkstelligen mag, viel Blut wird immer verloren gehen, da die Ligaturen das weiche Fruchtkuchengewebe und die dünne Sackwand leicht durchschneiden und deshalb wiederholt angelegt werden müssen. 